Artigo de Revisão

Hegemonia - Revista Eletrônica do Programa de Mestrado em Direitos Humanos, Cidadania

e Violência/Ciência Política do Centro Universitário Unieuro

ISSN: $1809-1261$

UNIEURO, Brasília, número 27 (Especial), 2019, pp. 58-73.

Recebido em: 17/9/2018

Avaliado em: 18/10/2018

Aprovado em: 14/11/2018

\title{
ASSISTÊNCIA A CRIANÇA E AO ADOLECENTE EM \\ DIFERENTES FASE DA LEUCEMIA: APRENDIZAGEM DE NOVOS COMPORTAMENTOS COMO IMPACTO FAMILIAR
}

\author{
Adasildo Carvalho da Silva ${ }^{1}$, Maria Fabiana de Castro ${ }^{2}$, Marilia Forte Gomes ${ }^{3}$, Marcella Lemos \\ Brettas Carneiro ${ }^{4}$
}

Resumo: Este trabalho tem como objetivo a busca de evidências a assistência à criança que tem diferentes fase da leucemia e dando ênfase no impacto familiar em sua dinâmica de vida relativa ao diagnóstico. Alguns registros de câncer no Brasil com base populacional, foi observado que o câncer infantil pode varia de $1 \%$ a 4,6\%. A pesquisa objetiva traçar mapeamento de produções científicas brasileiras da temática do câncer em crianças. Foi feita a seleção de artigos nas bases de dados SCIELO, LILACS e Periódicos CAPES, e Clinicaltrials, Web of Science, Science Direct. Trata-se de um estudo de revisão, com abordagem descritiva e qualitativa. Foram selecionados um total de 17 artigos, a região que mais se destaca foi a Sudeste. As áreas da ciência que se destacam neste tipo de pesquisa e sub-áreas da Enfermagem e Medicina. A ênfase da pesquisa foi a clínico-epidemiológico, seguida do sócio cultural. Verificando-se quanto é complexo o processo de adoecimento da criança com câncer que afeta não só a família, afetando também os profissionais envolvidos neste processo. A oncologia pediátrica configura um grande desafio para pesquisadores e suas equipes. Para averiguar o tipo de câncer e extensão da doença é permitido escolher o tratamento mais adequado, como recurso à quimioterapia, radioterapia e até mesmo o transplante de medula óssea. A leucemia é uma doença de caráter biológico tendo grandes repercussões a nível psicológico e social.

Palavras-Chave: Câncer; Leucemia; Oncologia; Quimioterapia; Radioterapia; Impacto familiar.

\footnotetext{
${ }^{1}$ Graduado em Tecnóloga em Radiologia pela Faculdade Juscelino Kubistchek, aluno especial no programa de pósgraduação em Engenharia Biomédica da Universidade de Brasília-UnB.

${ }^{2}$ Graduada em Engenharia de produção pela Estácio de Sá, aluna de mestrado no Programa de Pós-graduação em Engenharia Biomédica da Universidade de Brasília- UnB.

3 Doutorado em Demografia pela Universidade Federal de Minas Gerais e Professora no Programa de Pósgraduação em Engenharia Biomédica da Universidade de Brasília- UnB.

${ }_{4}^{4}$ Professora adjunto da Faculdade de Planaltina/UnB. Doutora em Biologia Molecular pela Universidade de Brasília. Pós-doutorado em Nanobiotecnologia pela Universidade de Brasília
} 
Artigo de Revisão

Hegemonia - Revista Eletrônica do Programa de Mestrado em Direitos Humanos, Cidadania

e Violência/Ciência Política do Centro Universitário Unieuro

ISSN: 1809-1261

UNIEURO, Brasília, número 27 (Especial), 2019, pp. 58-73.

Abstract: This work has been a home for the one who has different phase of leukemia and giving the main study in his dynamics of life with the diagnosis of diagnosis. Thus, based on population, it was observed that the level of childhood cancer ranges from $1 \%$ to $4.6 \%$. The research aimed at the mapping of Brazilian scientific production of the theme of cancer in children. A selection of articles was made in the databases LILACS, PEPSIC, SCIELO and PUBMED BRAZIL. This is a review study, with descriptive and qualitative. A total of 36 articles were selected, a region that most stands out for a Southeast. The areas of science that stand out in this type of research and sub-areas of Nursing and Medicine. The emphasis of the research was a clinical-epidemiological, followed by the cultural partner. A. The process of making decisions about the breastfeeding process in the child is not as important as the process. Pediatric oncology poses a major challenge for researchers and staff. The type of cancer and extent of disease are most sought after, the most appropriate treatment, the use of chemotherapy, radiation therapy and even bone marrow transplantation. Leukemia is a biological disease with great repercussions at the psychological and social level. Key Words: Cancer; Leukemia; Oncology; Chemotherapy; Radiotherapy; Family impact.

\section{INTRODUÇÃO}

O câncer no mundo representa entre $0,5 \%$ a 3\% entre crianças isso comparadas à uma população em geral. No Brasil, com dados adquiridos do registro de câncer de base populacional observa-se que o câncer infantil pode ter uma variação de 1\% a 4\% (INCA 2007). Os tipos mais frequentes de câncer em crianças são leucemias, tumores no sistema nervoso central e linfomas. Sendo a leucemia o mais comum entres crianças menores de 15 anos, tendo em destaque a leucemia linfocítica aguda (LLA), os tumores do sistema nervoso central os mais comuns são o astrocitoma e o meduloblastoma, predominante no sexo masculino, ocorre principalmente entre menores de 15 anos tendo um pico de idade de 10 anos que representa cerca de $20 \%$ dos tumores infantis. $\mathrm{O}$ terceiro tipo de neoplasias malignas pediátricas são os linfomas. (LOPES, 2000).

A leucemia Linfoide Aguda (LLA), é uma neoplasia maligna, onde caracteriza o acúmulo de células linfoides imaturas na medula óssea, o sintoma apresentado pelo paciente resulta grau variável de anemia, neutropenia, trombocitopenia e até mesmo infiltração dos tecidos por células leucêmicas. (ANASTASI; LARSON, 2003). Pesquisas apontam que a LLA é desconhecida é improvável que a modificação leucêmica seja resultante de evento único, mas sim do acúmulo de vários métodos que envolvem interações complicadas entre a susceptibilidade do hospedeiro, existe os danos secundários, à exposição por agentes químicos ou físicos e a possível herança genética (LEITE; Et. al 2007). Como às probabilidades atuais 
Artigo de Revisão

Hegemonia - Revista Eletrônica do Programa de Mestrado em Direitos Humanos, Cidadania e Violência/Ciência Política do Centro Universitário Unieuro

ISSN: 1809-1261

UNIEURO, Brasília, número 27 (Especial), 2019, pp. 58-73.

de cura, 70\% das crianças arremetidas de câncer podem ser curadas se diagnosticadas precocemente e medicadas em centros especializados (BRASIL, 2007). Os tratamentos atuais têm dois grandes objetivos: ampliar as taxas de sobrevida, minimizando os efeitos tardios do quadro clínico; e reintegrar a criança na sociedade com qualidade de vida (CAMARGO, 2007). A questão prognóstica de mortalidade torna-se visível os principais tipos de câncer que agridem a população pediátrica, sua prevalência, o quadro epidemiológico (sexo, idade, raça, entre outros), as taxas de cura, a estimação de sobrevida e a quantidade de óbitos. (MONTE, 2007).

Existem fatores que moderam os impactos da doença onde influenciam a vulnerabilidade do cuidador, como o tipo de suporte social, em características de ajuda instrumental e apoio, às buscas de informações e o tempo de cuidado dedicado a criança. Nesses casos, essas famílias apresentam um nível médio de funcionamento em termos de consolidação e relacionamentos ao crescimento pessoal e manutenção do sistema familiar (GUIMARÃES; ENUMO, 2015). Hoje existem preconceitos ligados à doença se tornando assim um fator desencadeador de sofrimento e, nesse sentido, a percepção e conscientização da possibilidade de morte da criança com leucemia podem facilitar diversos pensamentos no cotidiano da família. Os sentimentos vêm muito da religiosidade onde são evidenciados nos cuidadores de crianças com câncer, os quais se manifestam pelas fases obtidas da leucemia (AMADOR; Et. al, 2013).

Em estudo exploratório descritivo, foram reveladas as experiências e necessidades de membros de famílias que possuíam criança com câncer são de grande impacto. A família e a criança encaram problemas como longos períodos de hospitalização, reintegrações frequentes, terapêutica e agressiva, com sérios efeitos desagradáveis advindos do próprio tratamento, dificuldades pela separação dos membros da família durante as internações, interrupção das atividades diárias, limitações na compreensão do diagnóstico, desajuste financeiro, angústia, dor, sofrimento e o medo direto ou indireto da possibilidade de morte. O conjunto dos estudos descritos ilustra o impacto da criança com leucemia no sistema familiar de modo que proporcionam aos profissionais de saúde, ligados à oncologia pediátrica, mais conhecimentos e desafios adicionais enfrentados por estas famílias (NASCIMENTO; Et. al, 2005).

A radioterapia é uma importante modalidade terapêutica para muitas crianças e adolescentes com câncer, mas devido ao seu potencial para efeitos adversos agudos e crônicos, sua indicação deve ser muito criteriosa. A severidade dos efeitos adversos tem relação direta com a intensidade de dose e relação inversa com a idade. O enfermeiro deve ser capaz de reconhecer as reações adversas, tais como distúrbios gastrointestinais, mielosupressão e reações 
Artigo de Revisão

Hegemonia - Revista Eletrônica do Programa de Mestrado em Direitos Humanos, Cidadania

e Violência/Ciência Política do Centro Universitário Unieuro

ISSN: 1809-1261

UNIEURO, Brasília, número 27 (Especial), 2019, pp. 58-73.

de pele devidas à radioterapia. Normalmente, estes efeitos são reversíveis. Entre os efeitos tardios, quase sempre irreversíveis, estão os distúrbios de crescimento com possibilidade de deformidade dos ossos e tecidos moles, retardo mental devido à imaturidade do sistema neurológico, esterilidade e a possibilidade de dano em órgãos irradiados (DIAS, 2008).

A equipe de saúde tem como papel principal oferecer ao paciente segurança e confiança, visando os obstáculos que o paciente tem para tomar conduta correta e obter êxito em sua assistência. Necessita uma implementação e operacionalização de ações que caracterizem à sistematização do cuidado individual e integral ao paciente e a comunidade com medidas interventivas e promissoras para a capacitação à saúde (MATIAS, 2016).

A maneira em que a criança possa reagir à hospitalização depende da sua idade, da preparação para a internação, das experiências anteriores com doenças, do apoio familiar, da equipe médica e das suas condições emocionais. O prognóstico do câncer na criança e no adolescente é influenciado positivamente por estratégias de diagnóstico precoce e continuidade do cuidado por meio do tratamento adequado no tempo oportuno. Entretanto, como os sinais e os sintomas do câncer infantil são pouco específicos e similares aos de doenças benignas mais comuns na infância, isso pode dificultar a suspeita de uma neoplasia e, consequentemente, atrasar o diagnóstico da doença (SILVA, 2011). O foco deste artigo será a busca de evidências relacionada à assistência em criança com leucemia e os impactos na família, assim caracterizando a buscas em periódicos que contenha o assunto abordado. O processo de urbanização de maneira rápida vem tendo um estilo de vida igual aos de padrões de consumo, sabendo disso os cientistas notaram que isso podem ser os responsáveis pelas diversas alterações fisiológicas ocorridas no ser humano, onde causam uma doença na população. Várias doenças crônicas como o câncer, estão se acarretando na população brasileira e a nível mundial acompanhadas com o aumento da expectativa de vida. Em meio estas doenças destaca-se a leucemia um dos tipos de câncer que mais acomete as crianças, firmando-se mais comumente naquelas com idade de 2 a 5 anos, e menos comum naquelas com idade inferior a 15 anos, chegando a ser $70 \%$ dos casos diagnosticados em crianças e adolescentes.

Contudo o objetivo do artigo e declarar e buscar cada vez mais evidências para assistência em crianças com leucemia, visando o impacto da família onde estar envolvida diretamente na vida da criança acometida desta patologia. As divergências no entendimento que possuem acerca da doença e tratamento, cumprem a experiência na medicina resultando em 
Artigo de Revisão

Hegemonia - Revista Eletrônica do Programa de Mestrado em Direitos Humanos, Cidadania

e Violência/Ciência Política do Centro Universitário Unieuro

ISSN: 1809-1261

UNIEURO, Brasília, número 27 (Especial), 2019, pp. 58-73.

uma união da equipe de saúde aos familiares, enquanto que a situação de doença ajuda a sustentar uma aproximação entre eles, visando assim a assistência à criança.

\section{METODOLOGIA}

Trata-se de uma revisão de literatura. As etapas foram percorridas em identificação do tema e formulação da questão norteadora, estabelecendo os critérios para inclusão e exclusão de estudos, definindo as informações a serem extraídas dos estudos selecionados, dando prioridade a avaliação dos estudos incluídos na revisão e na categorização dos estudos e interpretação dos resultados apresentação da revisão. Para obtenção de bibliografia, referente ao assunto de interesse, foram realizadas buscas nas bases SCIELO, LILACS e Periódicos CAPES, Clinicaltrials, Web of Science, e Science Direct bem como, com busca manual das listas de referências dos artigos incluídos na revisão. As estratégias de busca empregadas foram utilizando-se as palavras-chaves: Câncer, leucemia, oncologia, quimioterapia, radioterapia. O processo de busca foi estipulado para documentos publicados nos últimos dez anos. O critério de inclusão baseou-se na ASSISTÊNCIA A CRIANÇA EM DIFERENTES FASE DA LEUCEMIA INFANTIL E O IMPACTO FAMILIAR. Utilizou-se, também, a ferramenta PRISMA (preferred reporting items for systematic reviews and meta-analyses) (MOHER et. al., 2015), para orientação da pesquisa e escrita do artigo de revisão, para elaboração das discussões acerca do tema proposto. A maior parte dos artigos selecionados foram publicados por enfermeiros, os demais eram publicações da área médica e de psicologia sendo um desses em contato com fisioterapia evidenciando assim que os pesquisadores da área da saúde têm se preocupado em publicar assuntos relacionados a leucemia e os impactos familiar, inferindo-se que há inquietação em investigar, compreender e assistir a criança no contexto familiar visando um diagnóstico como leucemia em suas diversas fases.

Em relação ao idioma, 10 artigos encontravam-se em inglês. Os estudos foram ainda observados em relação à abordagem da pesquisa e, assim, classificados quanto ao nível de evidência. A abordagem qualitativa foi utilizada em 5 artigos, 9 abordagens quantitativa. Os 17Artigos selecionados estavam distribuídos em periódicos distintos, onde houve grande concentração de publicação entre cuidadores de crianças com câncer, citados em periódicos específicos. Figura 1 relata a listagem dos artigos incluídos, bem como as características citadas respectivamente. 
Artigo de Revisão

Hegemonia - Revista Eletrônica do Programa de Mestrado em Direitos Humanos, Cidadania e Violência/Ciência Política do Centro Universitário Unieuro

ISSN: $1809-1261$

UNIEURO, Brasília, número 27 (Especial), 2019, pp. 58-73.

Figura 1, relato de bases, bibliografias, e resultados encontrado para construção do

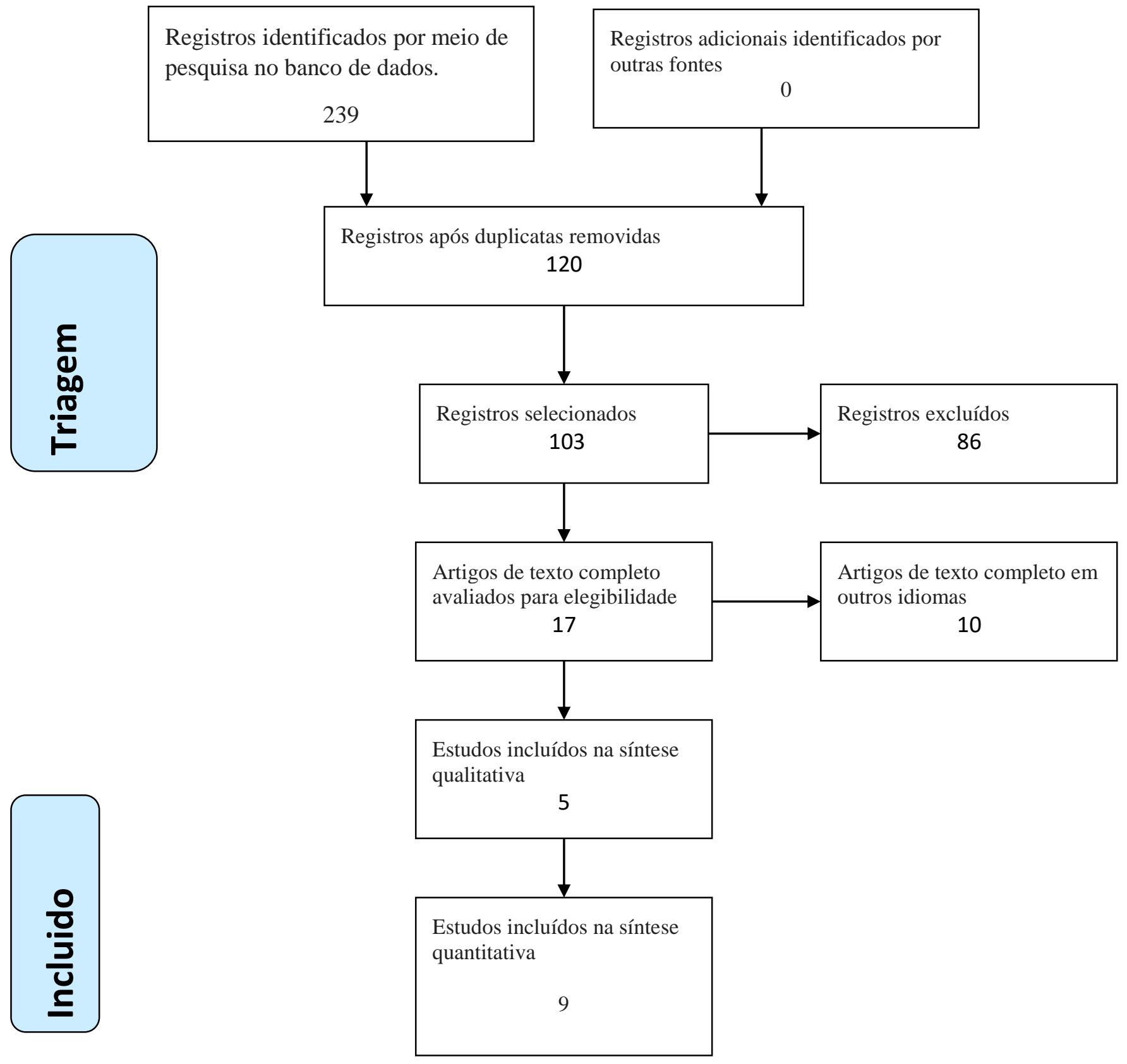


Artigo de Revisão

Hegemonia - Revista Eletrônica do Programa de Mestrado em Direitos Humanos, Cidadania

e Violência/Ciência Política do Centro Universitário Unieuro

ISSN: $1809-1261$

UNIEURO, Brasília, número 27 (Especial), 2019, pp. 58-73.

\section{DISCUSSÕES}

Determinados autores descreveram que biomateriais tiveram utilizações sugeridas como molde de enxerto de pele, agente cicatrizador, material para sutura, agente antimicrobiano, géis, cápsulas e soluções. Os Biomateriais brotados a partir do látex têm sido vistos como aceleradores de reparação tecidual e que oferecem a capacidade de acrescentar a produção da matriz extracelular. Os Biomateriais são substâncias ou concertos de substâncias sintéticas ou naturais, projetados para suprirem parte ou função orgânica de forma segura, econômica e aceitável fisiologicamente, atuando em contato direto com tecidos vivos e com sistemas biológicos podendo ser de uso temporário ou definitivo, os quais propor-se tratar ou substituir, parcial ou totalmente, qualquer tecido, órgão ou função do corpo (BERTOLINO, 2018).

Atualmente os biomateriais têm ganhado bastante atenção da comunidade científica no que se refere ao emprego tanto em saúde quanto em tecnologia. Eles vêm proporcionando grandioso sucesso em diversas aplicações, tais como o tratamento de feridas e doenças e, reparo ou substituição de órgãos e tecidos. Existem exemplos específicos que consistem em sua utilização como próteses ortopédicas, placas ósseas, cimento ósseo, ligamentos e tendões artificiais, implantes dentários, dispositivos de reparação da pele, próteses de vasos sanguíneos e diversos outros. Os biomateriais estão sendo considerados como materiais ou substâncias, diferentes de um fármaco, arquitetados para serem aplicados em um tecido, órgão ou ainda, para preencher alguma função no organismo, de uma forma geral, são utilizados em qualquer método ou propósito médico e/ou biomédico (MAGNO, 2013).

Outra aplicação para uso de látex foi o estudo experimental dos benefícios antiinflamatório que uma proteína, conhecida por "Hev b 13", retirada do látex natural de Hevea brasiliensis para tratamento de sepse, que é o quadro de doença infecciosa que responde há uma inflamação sistêmica grave (ARAÚJO. L. A; MELO-REIS P.R., MRUE F, 2017). A também a junção das potencialidades do látex com outros compostos de origem natural como a Papaína, Própolis e Calêndula para tratamento de feridas subcutâneas, em âmbito de pesquisa, demonstram melhora nos aspectos de cicatrização das feridas do órgão pele (XAVIER O., 2017). 
Artigo de Revisão

Hegemonia - Revista Eletrônica do Programa de Mestrado em Direitos Humanos, Cidadania

e Violência/Ciência Política do Centro Universitário Unieuro

ISSN: 1809-1261

UNIEURO, Brasília, número 27 (Especial), 2019, pp. 58-73.

Uma aplicação da utilização da Hevea Brasilienses nos Sistema Único de Saúde (SUS) é o diagnóstico artrite reumatoide, utilizando a técnica de látex, que é aglutinação do látex que são sensibilizadas por gamaglobulina humana (antígeno), que ao ser misturada ao soro de pacientes que possuem fatores reumatoides (FR) (OLIVEIRA, 2015). De acordo com anexo da tabela do SUS o valor é de 1,89 reais para sua realização. Pesquisadores vêm observando o poder cicatrizante que é uma defesa natural da planta, apresentando suas formas e vantagens. o látex tem seu fator de crescimento por uma composição que atua promovendo neovascularização e adesão celular (OLIVEIRA, 2008).

Além da utilização do látex para produção de luvas, sendo desenvolvidos estudos para controlar e diminuir aspectos asmagênico em aplicações da área da saúde para utilização desse insumo essencial de baixo custo para realização de procedimentos médicos (LaMontagne et al., 2006).

É apresentado na Figura 2 onde são as áreas que o Brasil tem investido de acordo com a natureza da pesquisa relacionando o número de pesquisas e valor investido para os setores em subáreas de aplicada / estratégica, básica, infraestrutura, não é classificável, outras ações de C\&T, saúde coletiva, tecnológica, translacional e não informado.

Dentre as classificações que o banco de dados do SUS de saúde informa, apresentadas na Figura 2 valores em reais por milhão, sendo elas de naturezas de aplicada / estratégica com número de pesquisa de 3449 e investimento de $\mathrm{R} \$ 537.829 .430,32$, para atenção básica pesquisa 679 e investimento de $\mathrm{R} \$ 132.978 .930,66$, infraestrutura 76 e investimento $\mathrm{R} \$ 57.128 .327,36$, não é classificável 541 e investimento $\mathrm{R} \$ 46.689 .629,57$, outras ações de C\&T 114 e investimento $\mathrm{R} \$ 63.481 .674,28$, saúde coletiva 305 e investimento $\mathrm{R} \$ 16.884 .560,23$, tecnológica 307 e investimento $\mathrm{R} \$ 49.937 .309,44$, translacional 13 de investimento $\mathrm{R} \$ 5.725 .033,81$, não informado 68 e investimento $\mathrm{R} \$ 27.642 .008,31$, Destacando-se a verba destinada ao setor tecnológico que o SUS aplica apoiando 348 pesquisas nesse setor e destinando o valor de R $\$ 170.402 .965,8$, em que está inserido os recursos de pesquisa para área de produtos de novas tecnologias para inserção no SUS para atendimento a pacientes que necessitam do sistema para tratamento, o que é benéfico pois representam futuras atualizações de tratamento que o país pode investir (Saúde, 2018). 
Artigo de Revisão

Hegemonia - Revista Eletrônica do Programa de Mestrado em Direitos Humanos, Cidadania e Violência/Ciência Política do Centro Universitário Unieuro

ISSN: 1809-1261

UNIEURO, Brasília, número 27 (Especial), 2019, pp. 58-73.

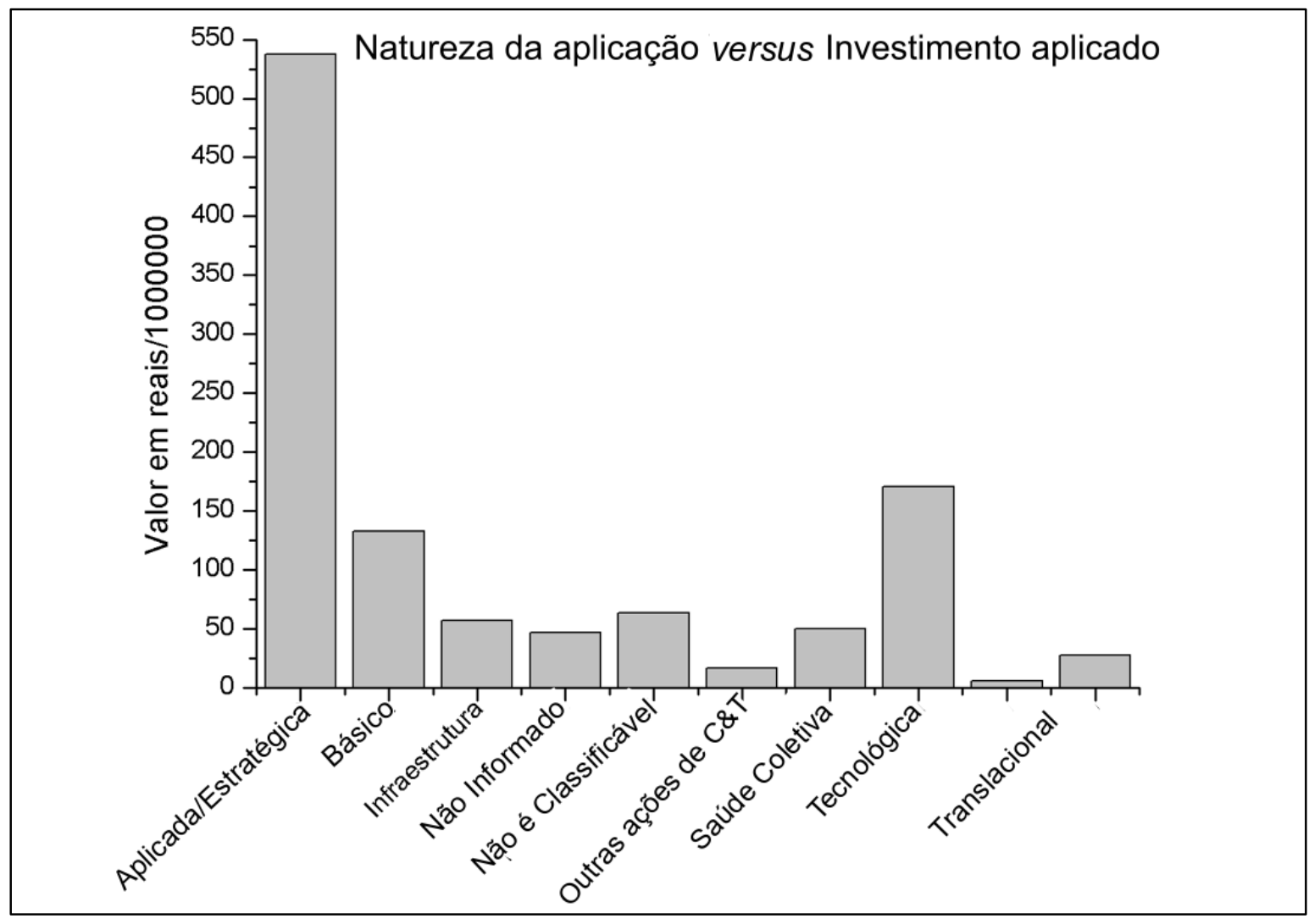

Figura 2 - Dados quantitativos da aplicação de recursos do SUS para o setor da pesquisa no Brasil, de acordo com a natureza da aplicação e valor a ser aplicado em cada subgrupo (Saúde, 2018).

$\mathrm{Na}$ Figura 3 é a relação entre as porcentagens de valores em cada setor, demonstrando que os recursos em suma são destinados a aplicação/estratégias com 74,69\%, atendimento básico de 14,71\% e Tecnologia com percentual de 7,54\%. As outras áreas os valores investidos estão em torno de $0,15 \%$ a $1,17 \%$. Demonstrando que o investimento do SUS em novas tecnologias é o terceiro setor que se tem preocupação para investimento em períodos de médio e longo prazo para se obtenção de resultados. 
Artigo de Revisão

Hegemonia - Revista Eletrônica do Programa de Mestrado em Direitos Humanos, Cidadania e Violência/Ciência Política do Centro Universitário Unieuro

ISSN: $1809-1261$

UNIEURO, Brasília, número 27 (Especial), 2019, pp. 58-73.

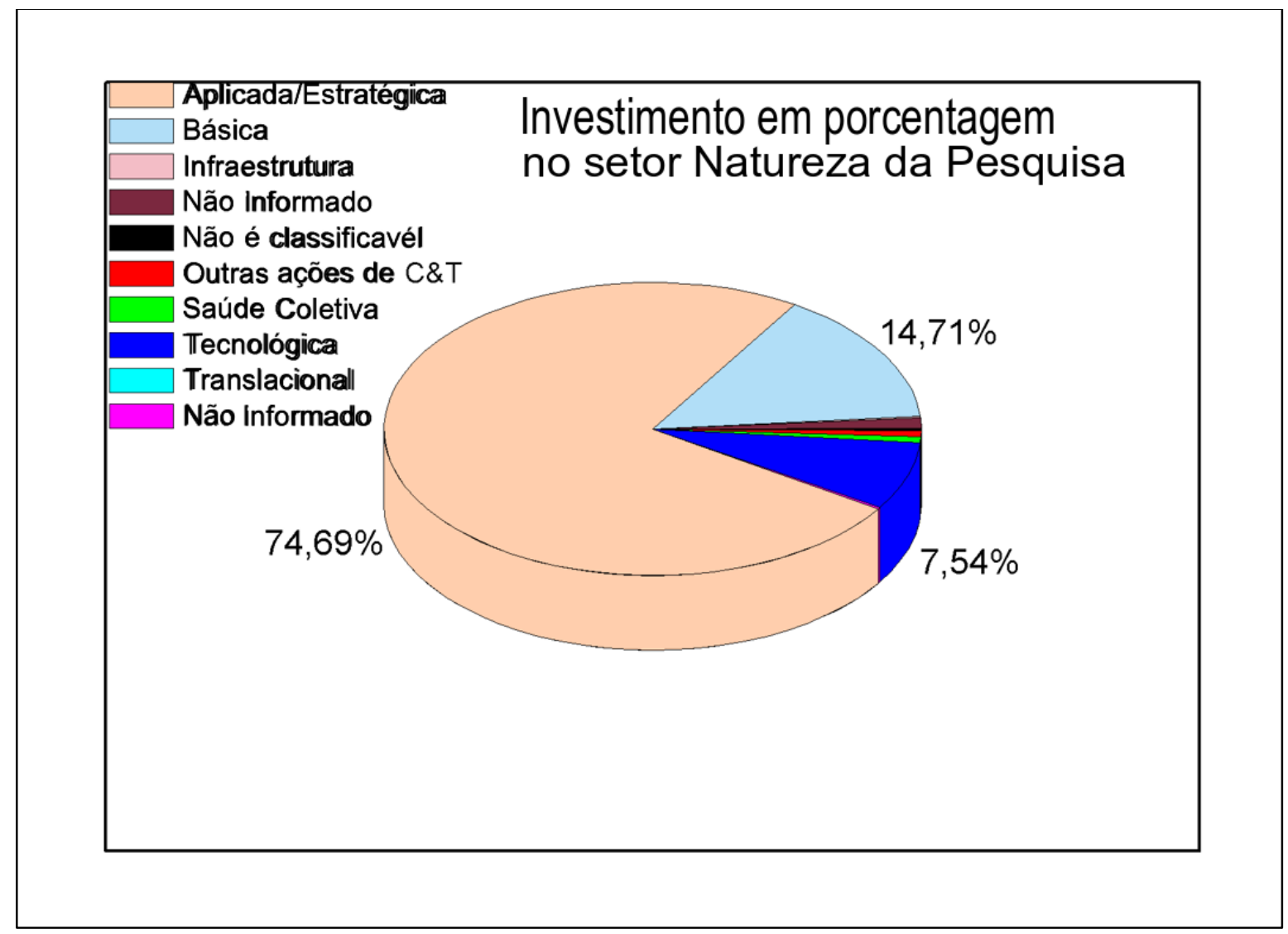

Figura 3- estimativa da quantidade de casos de leucemia em 2017 para crianças e adolescentes segundo estatuto nacional de câncer.

A Figura 3 temos a estimativa dos casos de leucemia para o ano de 2018, com 43,15\% para Região Sudeste seguido pela Região Nordeste com 22,87\%, Região Sul com 20,93\% e Regiões Centro-oeste e Norte de 6, 57\% e 6,48\% respectivamente. 
Artigo de Revisão

Hegemonia - Revista Eletrônica do Programa de Mestrado em Direitos Humanos, Cidadania e Violência/Ciência Política do Centro Universitário Unieuro

ISSN: 1809-1261

UNIEURO, Brasília, número 27 (Especial), 2019, pp. 58-73.

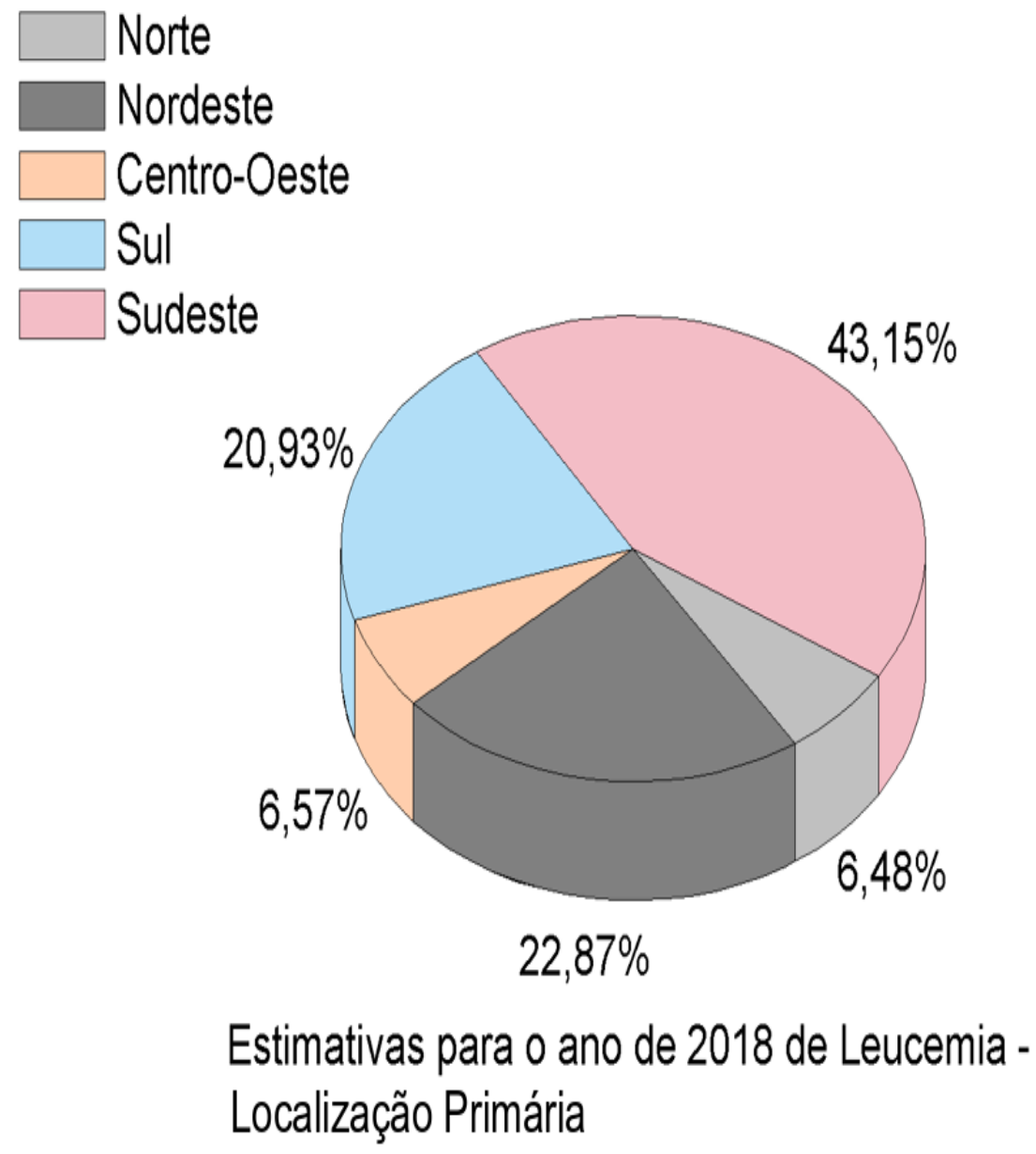

Figura 4 Estimativa para 2018 com casos de leucemia em localização primaria. Segundo instituto nacional de câncer.

Assim comparando os dados da Figura 2 e Figura 3 que são referentes aos anos de 2017 e 2018, temos uma redução de 4,87\% de números de casos para região Sudeste, para a Região Nordeste houve um acréscimo de 1,04\% na estimativa de casos de leucemia, já com a Região Sul foi de 10,45\% de acréscimo na estimativa, já o Norte tem-se o acréscimo de 13,27\% dos casos, a Região Centro-Oeste teve Redução de 3,51\% na estimativa da leucemia. 
Artigo de Revisão

Hegemonia - Revista Eletrônica do Programa de Mestrado em Direitos Humanos, Cidadania e Violência/Ciência Política do Centro Universitário Unieuro

ISSN: 1809-1261

UNIEURO, Brasília, número 27 (Especial), 2019, pp. 58-73.

A Figura 5 temos a estimativa de novos casos de leucemia para o ano de 2018 em relação ao gênero feminino e masculino de acordo com as regiões brasileiras, demonstrados em gráficos, para a Região Norte a estimativa para homens com leucemia é 390 casos, para mulheres é de 310, região centro-oeste 390 para homens e 320 para mulheres, região nordeste 1380 novos casos para homens e 1090 para mulheres, sudeste com 2500 para homens e 2160 para mulheres, já na região sul é estimado 1280 novos casos e 980 para mulheres.

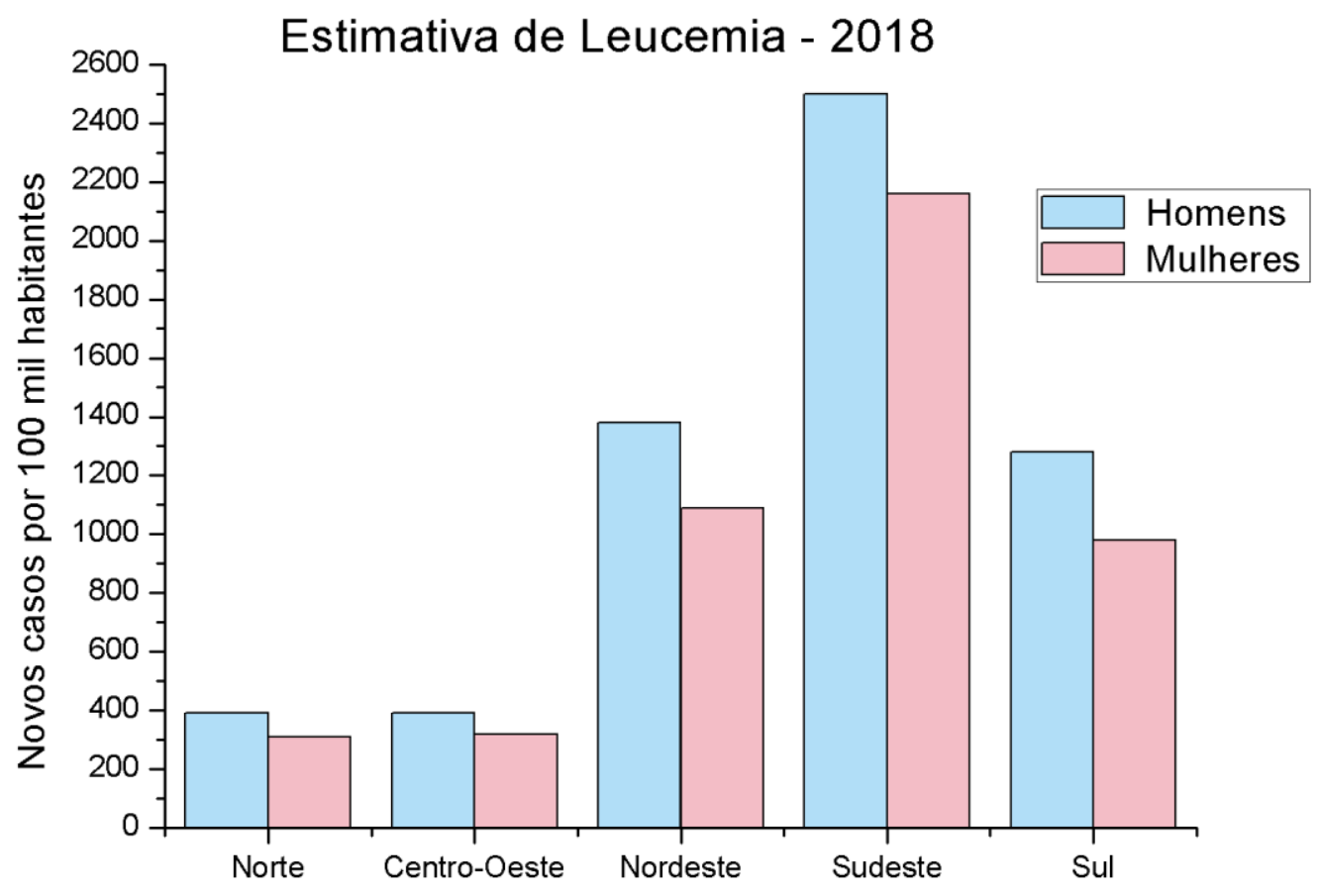

Figura 5 Estimativas de números de casos de câncer em 2018, por Região, em relação a homens e mulheres, segundo INCA.

\section{CONSIDERAÇÕES FINAIS}

O estudo das neoplasias malignas em crianças não é tão alto como em adultos, porém, no Brasil como em outros países, constitui-se uma as causas de morte mais frequente e, se eliminadas outras causas, como infecção e desnutrição, as neoplasias passam a constituir um problema de 
Artigo de Revisão

Hegemonia - Revista Eletrônica do Programa de Mestrado em Direitos Humanos, Cidadania

e Violência/Ciência Política do Centro Universitário Unieuro

ISSN: $1809-1261$

UNIEURO, Brasília, número 27 (Especial), 2019, pp. 58-73.

saúde, especialmente no grupo de 1 a 5 anos de idade. O câncer infantil atinge 10 em cada 100 mil crianças a cada ano em todo o mundo, sendo que uma criança em cada 600 pode desenvolvêlo durante a infância. $\mathrm{Na}$ atualidade $2 / 3$ destes cânceres podem ser considerados curáveis se a terapêutica instituída for adequada (LIMA, 1996).

Para Sousa; Escobar (2002) as crianças com patologia crônica e mais complexas necessitam de reintegrações mais frequentes, sendo que as mesmas são extensas ao iniciar o tratamento, muitas vezes com longos dias de internação. O tempo de internação depende muito da resposta da criança ao tratamento e da ausência de complicações.

Faz-se necessário que os profissionais, no contexto dos conhecimentos adquiridos estejam atentos às pesquisas referentes aos tipos de patologias e as formas como se apresentam para que cada ação seja destinada de acordo com cada característica apresentada.

Justifica-se o desenvolvimento desta pesquisa, pela grande permanência em hospitais destas crianças, sendo a equipe de saúde, principalmente a enfermagem, pode criar condições em um espaço lúdico onde a realidade vivenciada pela criança, no hospital, seja envolvida pelo imaginário, facilitando sua elaboração e aceitação.

O presente estudo mostrou que, diante da vivência do câncer infantil, não apenas os acometidos com a doença mais também o familiar tem sido vítima de tais evidências, e também se mostrou que que a assistência em crianças com leucemia estar cada vez mais vazia em busca de mais estudos.

\section{REFERÊNCIAS}

AMADOR. D.D; Et.al. Repercussões do câncer infantil para o cuidador familiar: revisão BRASIL. A Reprodução, total ou parcial, das informações. Ministério da Saúde Praça Cruz Vermelha, 23 - Centro - 20230-130 - Rio de Janeiro - RJ - INCA, 2018.

BRASIL. Instituto Nacional do Câncer (Brasil). Estimativa 2008: Incidência de câncer no Brasil. Rio de Janeiro (RJ): INCA; 2007.

BRASIL. Instituto Nacional do Câncer. Estimativa 2008: Incidência de câncer no Brasil. Rio de Janeiro (RJ): INCA; 2007. 
Artigo de Revisão

Hegemonia - Revista Eletrônica do Programa de Mestrado em Direitos Humanos, Cidadania e Violência/Ciência Política do Centro Universitário Unieuro

ISSN: 1809-1261

UNIEURO, Brasília, número 27 (Especial), 2019, pp. 58-73.

BRASIL.http://www.uespi.br/prop/XSIMPOSIO/TRABALHOS/PRODUCAO/Ciencias \%20da\%20Saude/LEUCEMIA\%20E\%20OS\%20CUIDADOS\%20DE\%20ENFERMAGE M\%20-\%20REVISAO\%20DE\%20LITERATURA Acessado em: 03 Ago. 2018.

DIAS, E. B. Leucemia na Infância. [2008]. Disponível em: <http://amigonerd.net/trabalho/40739-leucemia-na-infancia> Acesso em 01 Ago. 2018.

GUIMARÃES. A.A; ENUMO. S.R.F. Impacto familiar nas diferentes fases da leucemia infantil. Psicol. teor. prat. vol.17 no.3 São Paulo dez. 2015.

Integrativa. http://dx.doi.org/10.1590/S0034-71672013000200017 Rev. bras. enferm. vol.66 no.2 Brasília Mar./Apr. 2013.

KURACHIMA. C. B. Cuidados paliativos e oncologia pediátrica: o cuidar além do curar. São Paulo (SP): Lemar; 2007.

LEITE. E. P; Et. al. Fatores prognósticos em crianças e adolescentes com Leucemia Linfóide Aguda Rev. Bras. Saúde Matern. Infant., Recife, 7 (4): 413-421, out. / dez., 2007

LIMA, R. A. G; Et al. Assistência à criança com câncer: alguns elementos para a análise do processo de trabalho. Rev Esc Enf USP, v. 30, n. 1, p.14-24, abr 1996.

LOPES. C. B. Pediatria oncológica: noções fundamentais para o pediatra. São Paulo (SP): Lemar; 2000.

MATIAS. S.R. Assistência de enfermagem às crianças com leucemia linfoblástica aguda (LLA) Revisão Bibliográfica. Artigo apresentado no Curso de Enfermagem da Faculdade São Lucas como requisito para obtenção do título de Bacharel em Enfermagem. PORTO VELHO-RO.

MOHER D; Et al. The PRISMA Group. Preferred Reporting Items for Systematic Reviews and Meta-Analyses: The PRISMA Statement. PLoS Med 6(7): e1000097. doi:10.1371/journal. pmed1000097. 2009.

MONTE. O; Et.al. Carcinoma de tireóide na infância e adolescência. Arq Bras Endocrinol Metabol 2007; 51(5): 763-8.

NASCIMENTO. L.C; Et.aL. Crianças com câncer e suas famílias http://dx.doi.org/10.1590/S0080-62342005000400014 .Rev. esc. enferm. USP vol.39 no.4 São Paulo Dec. 2005. 
Artigo de Revisão

Hegemonia - Revista Eletrônica do Programa de Mestrado em Direitos Humanos, Cidadania e Violência/Ciência Política do Centro Universitário Unieuro

ISSN: 1809-1261

UNIEURO, Brasília, número 27 (Especial), 2019, pp. 58-73.

SILVA, L. R; At al. . Leucemia e os cuidados de enfermagem: revisão de literatura. 2010. SOUSA, V. B; ESCOBAR, E. M. A . Atuação do enfermeiro na assistência à criança com leucemia linfoblástica aguda. Rev Enferm UNISA, n.3, 2002, p. 8-12. 